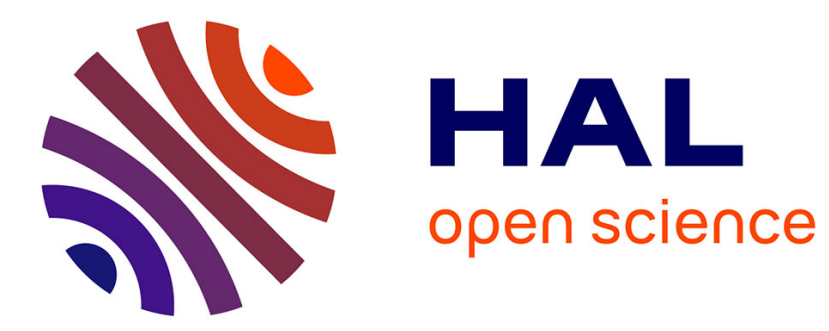

\title{
Jonctions Josephson à barrière semiconductrice
}

Ph. Cardinne, M. Marti, Maurice Renard

\section{To cite this version:}

Ph. Cardinne, M. Marti, Maurice Renard. Jonctions Josephson à barrière semiconductrice. Revue de Physique Appliquée, 1971, 6 (4), pp.547-550. 10.1051/rphysap:0197100604054700 . jpa-00243586

\section{HAL Id: jpa-00243586 https://hal.science/jpa-00243586}

Submitted on 1 Jan 1971

HAL is a multi-disciplinary open access archive for the deposit and dissemination of scientific research documents, whether they are published or not. The documents may come from teaching and research institutions in France or abroad, or from public or private research centers.
L'archive ouverte pluridisciplinaire HAL, est destinée au dépôt et à la diffusion de documents scientifiques de niveau recherche, publiés ou non, émanant des établissements d'enseignement et de recherche français ou étrangers, des laboratoires publics ou privés. 


\title{
JONCTIONS JOSEPHSON A BARRIĒRE SEMICONDUCTRICE
}

\author{
Ph. CARDINNE $\left(^{*}\right)$, M. MARTI et M. RENARD
}

\author{
C.E.C. L'Air Liquide, Centre de Recherches, 38-Sassennage*
}

\begin{abstract}
Résumé. - Les effets Josephson continu et alternatif ont été observés sur des jonctions planes pour lesquelles la barrière est constituée d'un film mince (100 à $200 \AA)$ semiconducteur obtenu par évaporation.

La variation de la résistance de jonction $R_{\mathfrak{f}}$ en fonction de la température est expliquée qualitativement par un modèle de bande simple. Des valeurs du gap supraconducteur $2 \Delta$ différentes de celles du plomb ont été observées.
\end{abstract}

Abstract. - The DC and AC Josephson effects are observed in some plane junctions where the barrier is a thin film (100 to $200 \AA)$ of semiconducting evaporated material.

Variations of the junction resistance $R_{\mathrm{j}}$ as a function of temperature are qualitatively explained by a simple band model. Values of superconductor gap $2 \Delta$ are observed to be different of the lead value.

L'idée de départ consiste à utiliser comme barrière tunnel entre deux supraconducteurs, non pas de l'oxyde comme on le fait habituellement, mais une substance qui présente une bande interdite de hauteur plus faible. C'est ainsi qu'au lieu d'utiliser une barrière de quelques $\mathrm{eV}$, ce qui nécessite pour l'observation de l'effet Josephson une épaisseur d'une vingtaine d'angstrams, nous avons cherché un corps semiconducteur qui puisse présenter une barrière de quelques dizaines de meV de haut, ce qui permet d'utiliser la technique facile d'évaporation avec des épaisseurs de 100 à $200 \AA$. L'intérêt en est évidemment la possibilité d'avoir une vitesse de phase des ondes électromagnétiques dans la barrière plus proche de celle de la vitesse de la lumière.

Disons quelques mots du modèle qui permet d'arriver à un tel résultat. Dans le cas d'un contact MétalSemiconducteur la position de la bande interdite du semiconducteur est fixée (dans la plupart des cas) par les valeurs comparées des potentiels d'extraction des constituants.

Comme nous le verrons par la suite, la condition pour que l'on puisse arriver à un fonctionnement Josephson est que la bande interdite tombe à l'extérieur du niveau de Fermi du métal ce qui se traduit par les deux figures :

Les situations représentées sur les figures ne peuvent subsister car il va apparaître, dans le cas 1 par exemple, des charges d'espaces négatives qui tendent à remplir la bande de conduction, ce qui va courber les bandes interdites pour arriver à cette situation qui mènerait d'ailleurs à un court-circuit à $T=0^{\circ}$ et en l'absence d'impuretés.
- Nous savons que l'épaisseur du semi-conducteur est suffisamment faible pour que le niveau de Fermi soit dans le prolongement de celui des métaux. Introduisons maintenant l'existence d'impuretés. Dans le cas du tellure il existe un niveau accepteur situé près de la bande de valence. Ainsi nous imposons au niveau de Fermi d'être artificiellement relevé et apparaîtront des charges localisées qui ne seront pas compensées, donc amèneront une courbure des bandes.

- D'autres charges vont jouer un rôle identique : ce sont les électrons thermiquement excités ; qualitativement on voit que plus le taux d'impuretés est grand et plus la hauteur de la barrière va être grande.

- Nous avons tenté de calculer donc la hauteur de cette barrière en fonction de la température et du taux d'impuretés et nous comparerons ce modèle à l'expérience.

Le principe du calcul est le suivant : soit $g(E)$ la densité d'état, nous écrivons que la densité électronique $\rho$ en un point $x$ est de la forme :

$$
\rho=\int_{0}^{\infty} \frac{\sqrt{E} \mathrm{~d} E}{1+\exp (E+e V / K T)}(g(E) \propto \sqrt{E}) .
$$

A cette densité qui est régie par la distribution de Fermi nous ajoutons la densité d'impureté $\rho_{0}$. Ainsi nous introduisons une courbure que l'on peut calculer en écrivant l'équation de Poisson.

$$
\frac{\mathrm{d}^{2} e V}{\mathrm{~d} x^{2}}=\frac{4 \pi}{\varepsilon} \int_{0}^{\infty} \frac{\sqrt{E} \mathrm{~d} E}{1+\exp (t+e V / K T)}+\frac{4 \pi}{\varepsilon} \rho_{0} .
$$

Nous avons deux variables indépendantes $E$ et $V$. Sans entrer dans le détail du calcul qui ne peut être 
fait analytiquement et nécessite l'emploi d'un calculateur, nous pouvons dire que le résultat final se présente sous la forme d'un réseau de courbes représentant une certaine fonction Izm en fonction de la hauteur maximale de la barrière $Z_{m}$.Izm ne dépend que des constantes du semiconducteur (masse effective-Cte diélectrique) (1). Le paramètre variable du réseau est un paramètre $\mathrm{A}$ qui dépend de la température et du taux d'impuretés. $I z$ et le paramètre $\mathrm{A}$ dépendant tous les deux de la température ceci permet d'utiliser le réseau pour étudier $Z_{m}$ en fonction de $T$ (cf. Fig. 1).

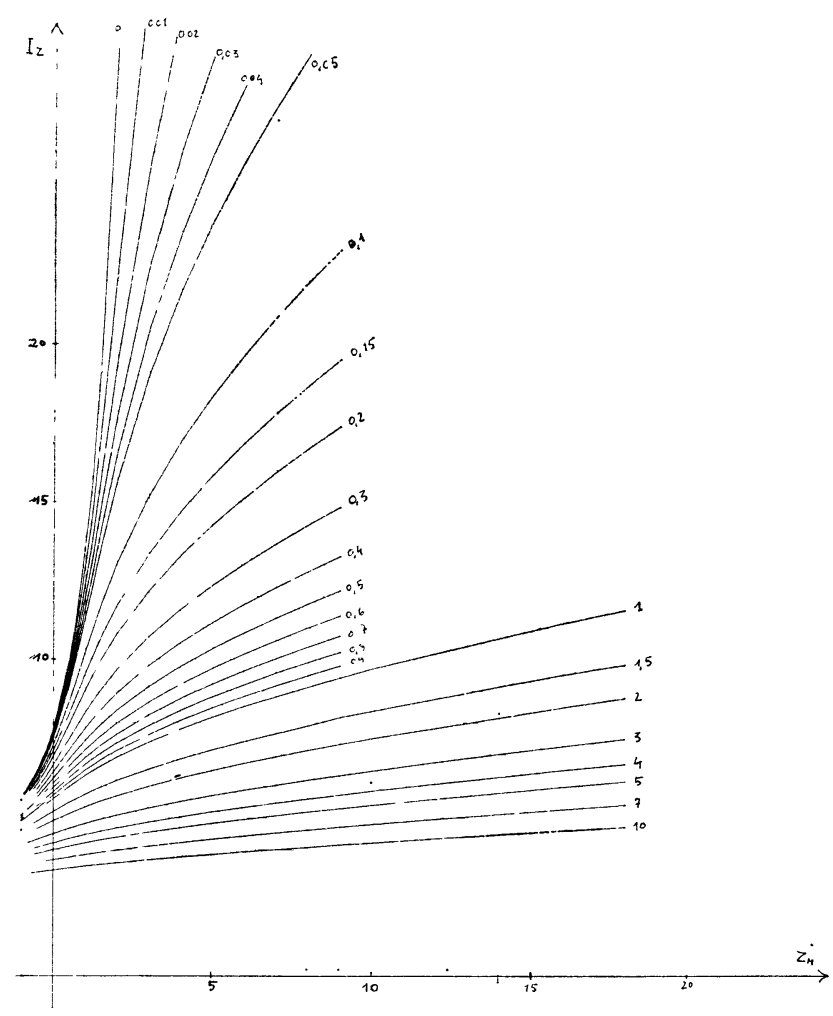

FIG. 1.

Résultats. Comparaisons. - Nous avons obtenu expérimentalement deux types de variation de la réssitance tunnel en fonction de la température. Le premier, très fréquent (Fig. 2), montre l'existence d'un maximum (les courbes successives proviennent précisément d'une diminution dans le dopage en fonction des cyclages, cette diminution étant due, sans doute, à une accumulation de dislocations dans la barrière).

Les courbes obtenues par le calcul ont qualitativement la même allure pour de faibles dopages (Fig. 3), la redescente de la résistance en fonction de la température n'est pas donnée par le modèle ; ceci est normal : en effet, l'excitation thermique arrive à faire passer les électrons au-dessus de la barrière ce qui explique la redécroissance de la résistance à haute température $T=110^{\circ} \mathrm{K}, K T \simeq 10 \mathrm{meV}$.

Le deuxième type de variation, beaucoup plus rarement observé, est représenté figure 4 ; la résistance décroît constamment avec la température. Les courbes obtenues par le calcul (Fig. 5) peuvent rendre compte

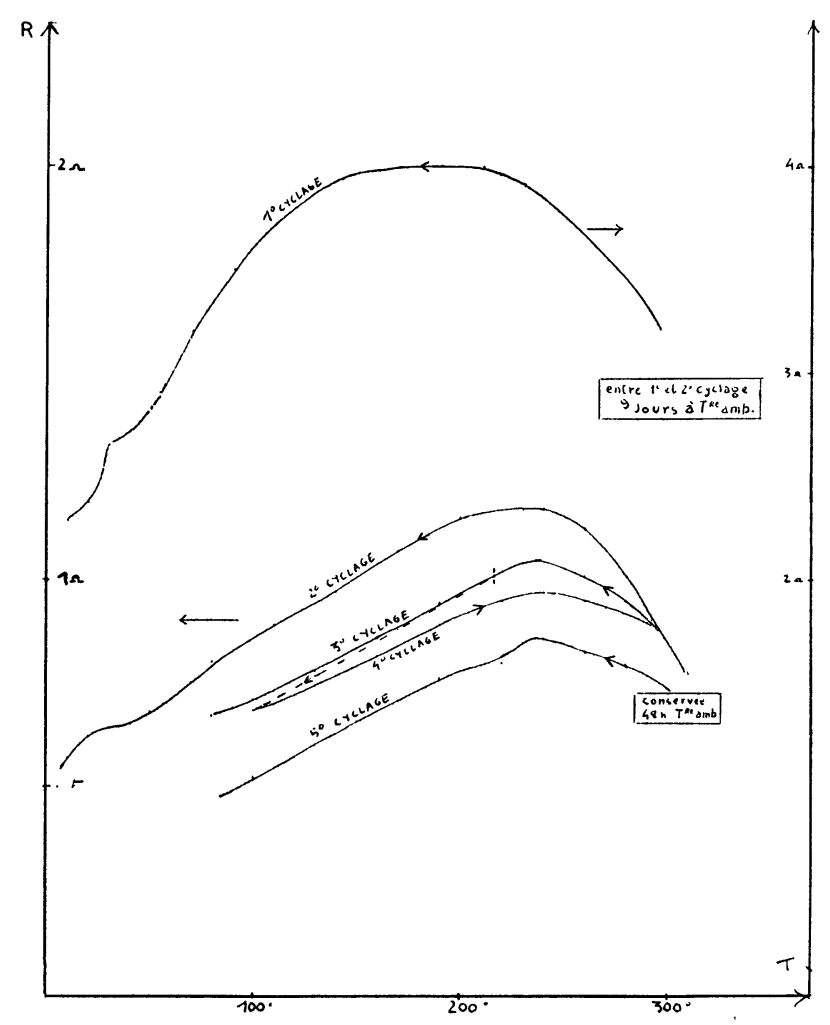

FIG. 2.

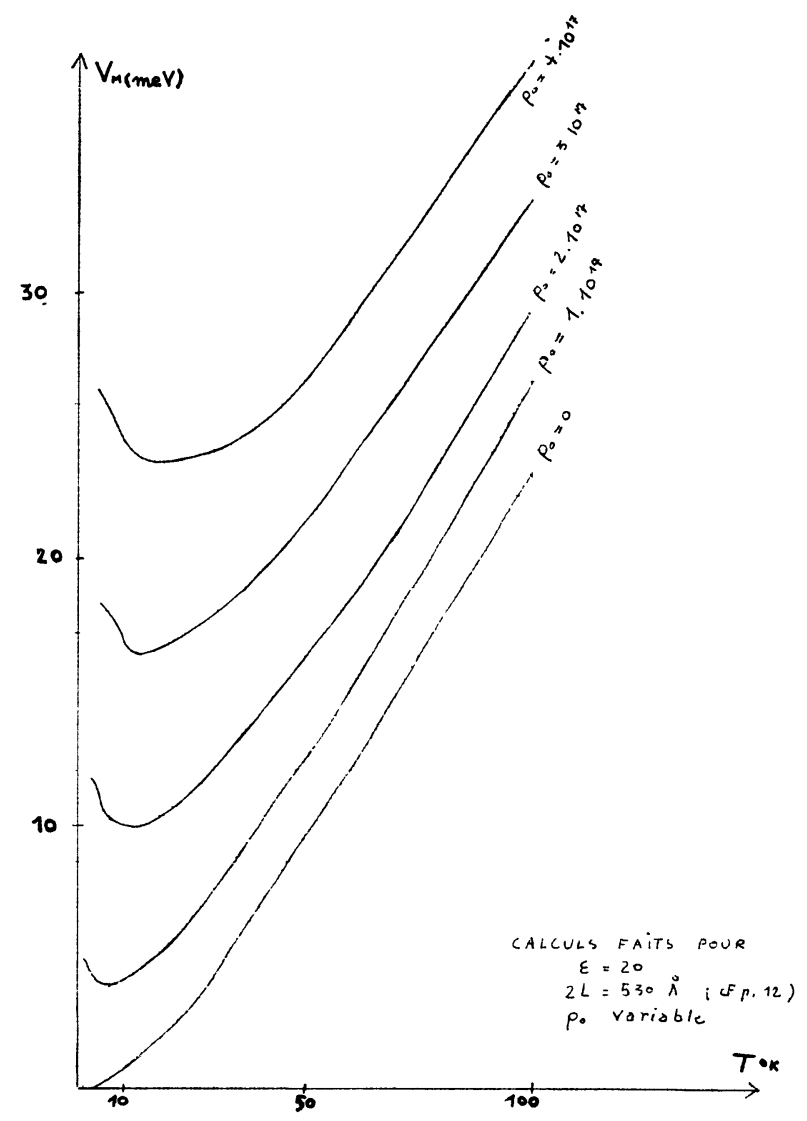

Frg. 3. 


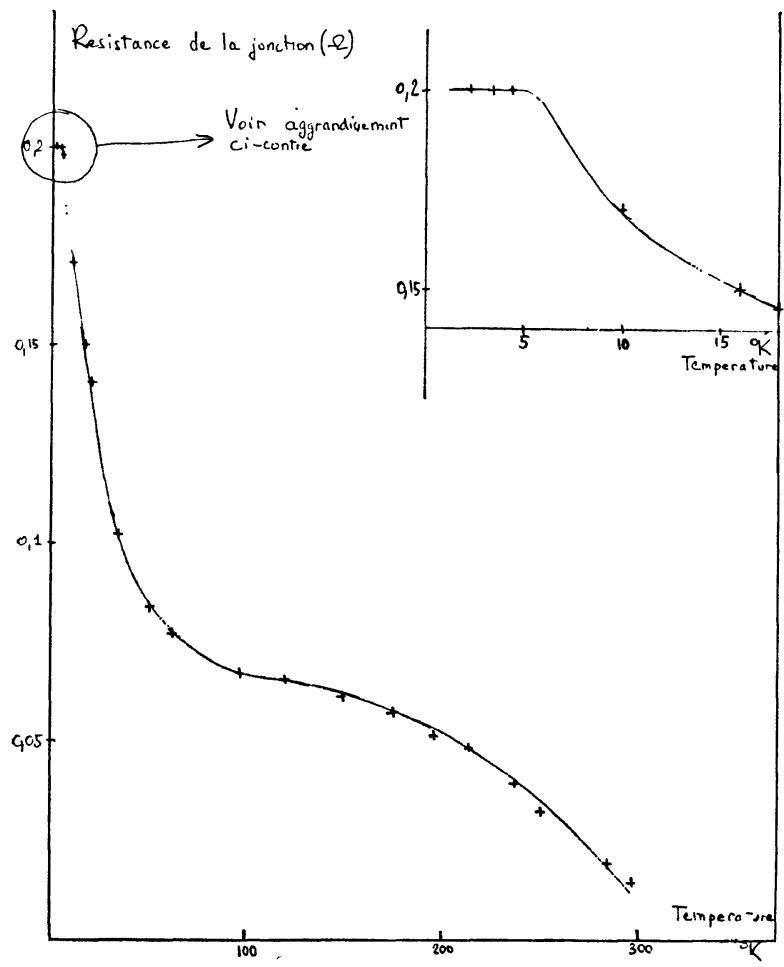

FIG. 4.

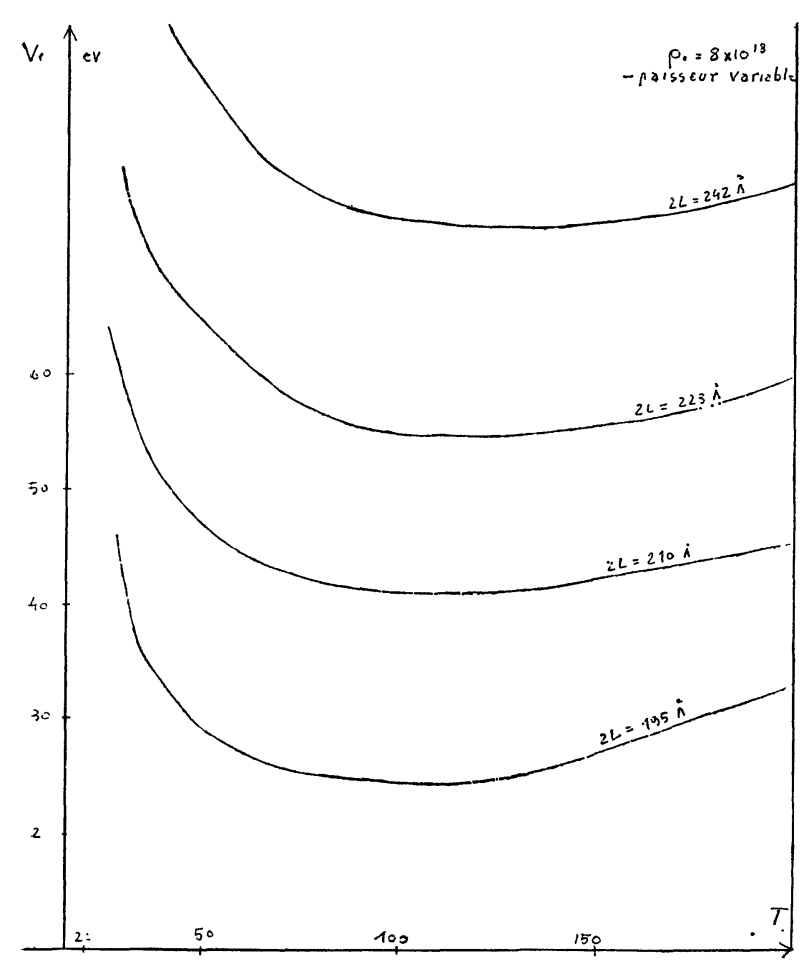

FIG. 5.

de ce comportement pour des valeurs plus élevées du dopage. La seule partie représentative est la partie de gauche. Pour les mêmes raisons que précédemment, la conduction directe devient prépondérante, le $k T$ étant du même ordre que la hauteur de la barrière.
Observations Josephson. - D'une manière générale, les jonctions ainsi élaborées présentent, outre l'effet Josephson, de l'effet Giaever qui caractérise une barrière tunnel. Nous voyons (Fig. 6) deux courbes caractéristiques pour deux jonctions d'épaisseur différente. La partie de conduction directe est plus grande pour la plus épaisse.

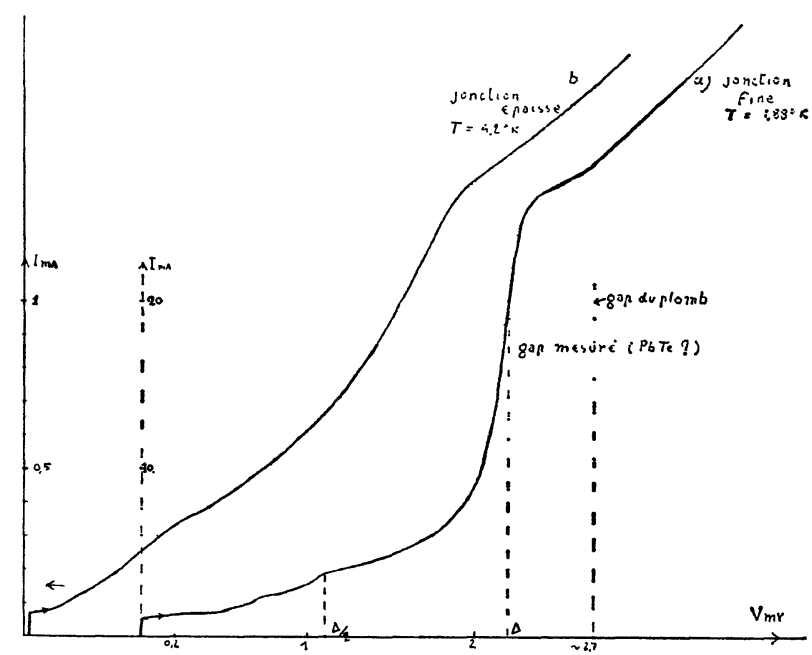

FIG. 6.

Ceci se traduit par le fait que le produit $R I c$, même dans le cas de la jonction la plus fine, est toujours inférieur à la valeur calculée par Ambegaokar et Baratoff

$$
\left(R_{\Omega} \times \mathrm{I}_{\mathrm{c} \mathrm{mA}} \simeq 1,9\right) .
$$

Ceci revient à dire qu'il existe une conduction ne contribuant pas au courant critique. Une autre observation intéressante est la mise en évidence de la formation à l'interface d'un composé $\mathrm{PbTe}$, ceci à partir de l'examen de la caractéristique Giaever. En effet, nous constatons :

1) que la valeur du GAP est nettement inférieure à celle du plomb, à peu près 2,3 contre $2,7 \mathrm{MeV}$,

2) que la variation du GAP en fonction de la température suit bien B. C. S.,

3) que le courant critique et le GAP s'annulent pour une valeur très peu différente de celle du plomb.

Interprétation. - La première idée qui vient à l'esprit est que le composé $\mathrm{PbTe}$ devient supraconducteur avec des caractéristiques différentes. A l'heure actuelle, ceci est discuté dans la littérature. Il est possible qu'une augmentation de la densité électronique suffise à rendre ce matériau supra. Ce phénomène aurait lieu dans les poches d'électrons situées près de l'interface.

D'autres explications sont possibles ; ainsi un effet de proximité n'est pas à exclure.

Courants critiques. - Les courants critiques des jonctions obtenues vont de $10 \mu \mathrm{A}$ à $300 \mathrm{~mA}$ suivant les conditions expérimentales. 
La fiabilité de ce type de jonction est assez bonne puisque nous avons conservé des jonctions à la température ambiante pendant une période de 8 mois. Les courants critiques après ce laps de temps avaient augmenté d'un facteur 3,5 .

Comportement magnétique. - Nous avons retrouvé le comportement magnétique classique des jonctions étroites et larges.

On appelle jonctions étroites les jonctions pour lesquelles la largeur est plus petite ou égale à $\lambda_{j}$, profondeur de pénétration Josephson $\left(W \leqslant \lambda_{\mathrm{j}}\right)$. Cette grandeur $\lambda_{j}^{2}$ est inversement proportionnelle à la densité de courant dans la jonction, donc liée aux propriétés tunnel de la barrière. Dans le cas des jonctions étroites (Fig. 7) nous retrouvons la courbe de diffraction classique de Fraunhoffer. $I_{c}$ s'annule chaque fois qu'il rentre un quantum de flux $\Phi_{0}$.

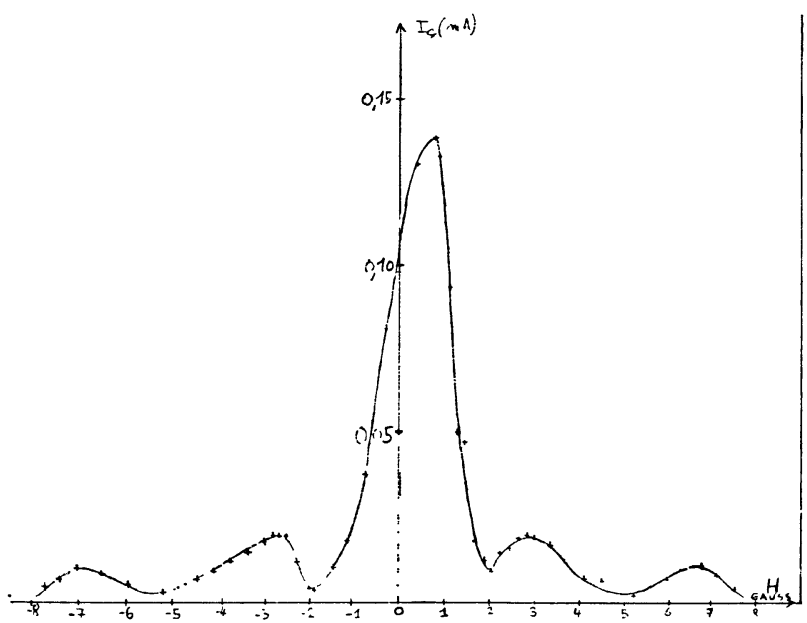

FIG. 7.

Dans le cas des jonctions larges $\left(W>\lambda_{\mathrm{j}}\right)$ la courbe $I_{\mathrm{c}}(H)$ a un aspect différent (cf. Fig. 7). Elle est inclinée par rapport à l'axe des intensités. Ceci a été observé dans la littérature et est lié à l'influence du «self champ » créé par le courant tunnel lorsque celui-ci est important.

Dans le cas, le courant ne passe plus d'une façon uniforme mais sur les bords de la jonction suivant une distance caractéristique d'écrantage $\lambda_{\mathbf{j}}$.

Le champ extérieur pour lequel le courant s'annule correspond alors au fait qu'il existe dans la jonction un nombre entier de vortex. Ainsi, dans le cas de la figure ci-après $n=1$ vortex et le champ extérieur est alors égal à :

$$
H_{\mathrm{c} 1}=\frac{\Phi_{0}}{2 \pi \lambda_{\mathrm{j}}(2 \lambda+a)} .
$$

Par ailleurs, le courant critique dans ce cas-là est égal à $I_{\text {tot. }}=2 L \lambda_{\mathrm{j}} \mathrm{j}_{1}$ pour les jonctions en croix. Si on reporte cette valeur dans la relation donnant $\lambda_{v}$ et si on élimine ensuite $\lambda_{\mathbf{j}}$ on arrive à une relation qui, mise en unités pratiques, est de la forme :

$$
I_{0 \mathrm{~mA}}=\frac{10^{4}}{2 \pi} H_{\mathrm{c} 1 \text { gauss }} \times L_{\mathrm{cm}}
$$

L étant la dimension parallèle au champ.

La figure 8 montre que les points expérimentaux correspondant aux diverses jonctions se placent correctement sur cette droite.

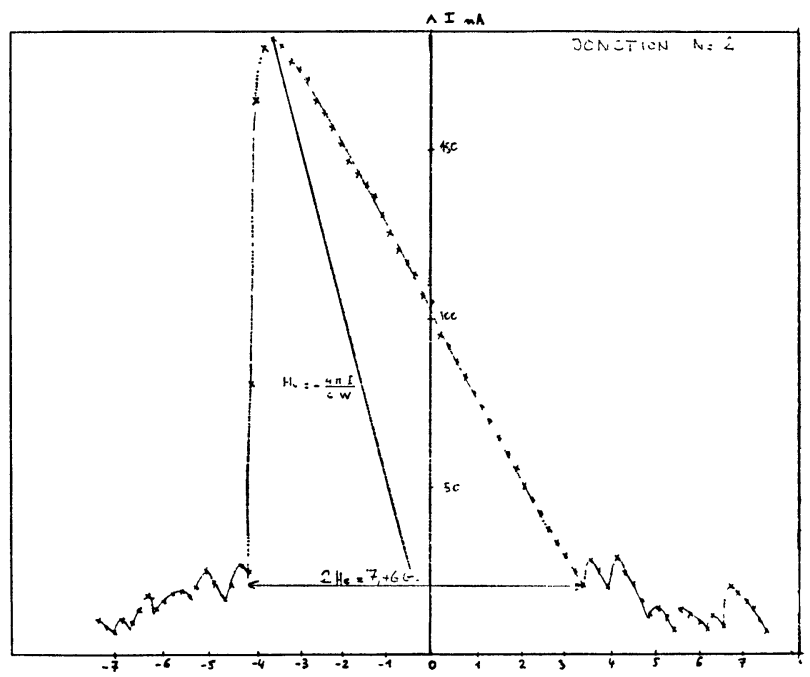

Fig. 8.

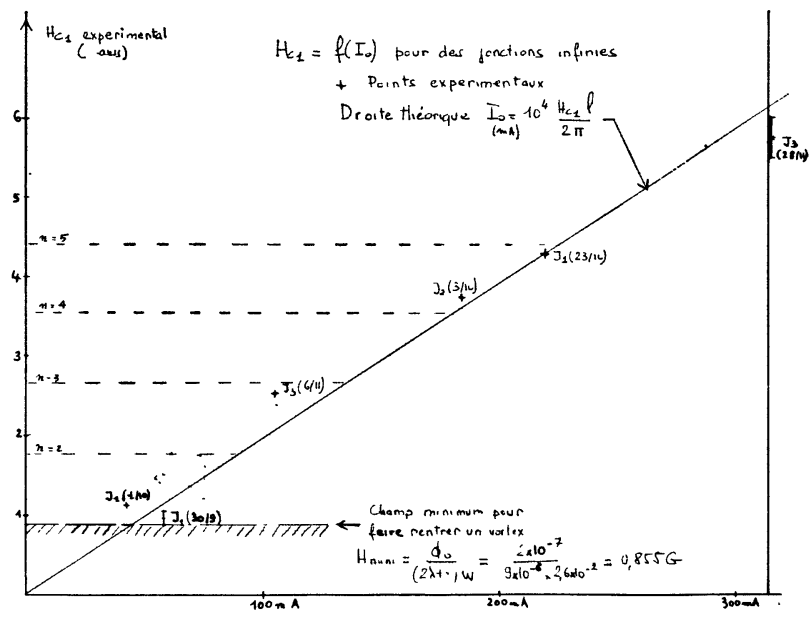

FIG. 9.

Applications. - Ce type de jonction a été irradié en guide d'onde à une fréquence voisine de $10 \mathrm{KMcs}$, ceci dans les laboratoires du L. C. I. E. (M. Pech).

Les steps induits ont été observés jusqu'au dixième step. Dans certains cas la détection de la $20^{\mathrm{e}}$ marche était également possible.

Le but poursuivi par le L. C. I. E. est la création d'un étalon de tension dont la précision serait, en gros, proportionnelle au nombre de marches détectables. 